\title{
Representación basada en caminos para la resolución simultánea del batching y scheduling
}

\section{Path based representation for simultaneous resolution of batching and scheduling}

Presentación: 6-7/10/2020

\section{Doctorando:}

\section{Sergio Ackermann}

INGAR - Instituto de Desarrollo y Diseño, Facultad Regional Santa Fe, Universidad Tecnológica Nacional - Argentina sackermann@santafe-conicet.gov.ar

\section{Director/a:}

\section{Jorge Marcelo Montagna}

\section{Co-director/a:}

\section{Yanina Fumero}

\section{Resumen}

El scheduling de la producción tiene un rol fundamental para lograr la operación eficiente de una planta batch multiproducto. La dificultad del problema y número de variantes que se pueden presentar ha hecho del mismo un área de constante investigación y desarrollo de modelos y estrategias de resolución. Usualmente el scheduling ha sido resuelto de manera simplificada, teniendo como datos el número y tamaño de batches (o lotes) necesarios para satisfacer la demanda. Sin embargo, la resolución simultánea del batching y scheduling lleva en general a la obtención de mejores soluciones. Los modelos desarrollados hasta el momento para abordar dichos problemas de manera simultánea son de una gran complejidad computacional, debido a su naturaleza combinatoria, por lo que lograr el desarrollo de modelos eficientes que permitan abordar problemas de gran tamaño continúa siendo un desafío. En este trabajo se presenta un novedoso modelo de programación mixta entera lineal (MILP) para la resolución simultánea del batching y scheduling en una planta batch multietapa, con unidades distintas operando en paralelo y fuera de fase en cada etapa.

Palabras clave: Planta batch multiproducto, batching, scheduling, tiempos discretos

\begin{abstract}
Production scheduling plays a fundamental role in achieving the efficient operation of a multiproduct batch plant. The difficulty of the problem and the number of variants that can be presented has made it an area of constant research, development of models and resolution strategies. Usually the scheduling problem has been solved in a simplified way, having as data the number and size of batches necessary to satisfy the demand. However, the simultaneous resolution of batching and scheduling generally leads to better solutions. The models developed to address these problems simultaneously are highly computationally complex, due to their combinatorial nature, so achieving the development of efficient models that allows addressing large problems continues to be a challenge. In this work is presented a new mixed-integer linear model (MILP) for the simultaneous
\end{abstract}


resolution of batching and scheduling in a multistage batch plant, with different units operating in parallel and out of phase in each stage.

Keywords: Multiproduct batch plant, batching, scheduling, discrete times

\section{Introducción}

En la industria de procesos, tienen un rol destacado las plantas de producción batch debido a la flexibilidad de las mismas para procesar varios productos y en diferentes cantidades utilizando el mismo equipamiento. En este tipo de plantas el scheduling de la producción juega un papel fundamental para lograr un funcionamiento eficiente. Numerosos trabajos han abordado dicho problema durante las últimas tres décadas, cubriendo aspectos teóricos, diferentes representaciones y métodos de solución, cuyo alcance ha sido detallado en exhaustivos reviews (Méndez y col. (2006), Maravelias (2012), Harjunkoski y col. (2014), Castro y col. (2018)).

En ambientes de producción secuencial (plantas flowshop), la mayoría de los modelos presentados en la literatura resuelven en una primera etapa el problema de "batching", es decir, determinan el número y tamaño de los batches necesarios para satisfacer las órdenes y la solución de ese problema es usada en una segunda etapa como dato en el problema de scheduling, el cual consiste entonces en determinar la asignación, secuenciamiento y los tiempos de inicio y fin de la operación de los lotes que se deben procesar. Las soluciones obtenidas resolviendo el problema en dos fases pueden resultar muy inferiores en comparación a las que se obtienen al resolver ambos problemas de manera simultánea, especialmente en el caso de plantas multiproducto que cuentan con unidades diferentes operando en paralelo y fuera de fase en cada etapa. Prasad y Maravelias (2008) han presentado el primer enfoque que considera la optimización simultánea de ambas decisiones, y desde entonces algunos enfoques en esta área, con ciertas limitaciones y características, han sido presentados (Sundaramoorthy y Maravelias (2008a), Sundaramoorthy y Maravelias (2008b)). Más recientemente, Fumero y col. (2014) han desarrollado un modelo de programación mixta-entera lineal (MILP) basado en slots para optimizar simultáneamente las decisiones de batching y scheduling para una planta batch multiproducto multietapa que opera a través de campañas de producción mixtas. Los trabajos mencionados anteriormente, adoptan una representación continua del tiempo. Un primer modelo de tiempo discreto que integra las decisiones de batching y scheduling en plantas secuenciales ha sido propuesto por Sundaramoorthy y col. (2009) y estudios recientes han mostrado que los modelos de tiempo discreto son más efectivos, desde el punto de vista computacional, para problemas de gran escala (Merchan y col. (2016)).

Sobre ambientes de producción secuencial, pero más generales, como es el caso de plantas batch multipropósito, Lee y Maravelias (2017a) han desarrollado modelos de scheduling con representación de tiempo discreto, considerando almacenamiento intermedio finito. Posteriormente, estos autores propusieron formulaciones MILP de tiempo discreto para optimizar simultáneamente las decisiones de batching y scheduling en este contexto, introduciendo un mecanismo de agrupación de unidades en conjuntos que son capaces de procesar cierto rango de tamaños posibles de los batches (Lee y Maravelias (2017b)).

\section{Desarrollo}

En este trabajo se aborda la solución simultánea de los problemas de batching y scheduling para el caso de una planta de producción batch multiproducto, compuesta por múltiples etapas, donde cada una de ellas cuenta con varias unidades de diferentes tamaños operando en paralelo y fuera de fase. La planta debe procesar un conjunto de órdenes de tamaño conocido, las cuales pueden ser satisfechas por uno o varios batches de diferentes tamaños. Cada orden monoproducto tiene asociada una fecha de emisión a partir de la cual puede comenzar a procesarse y otra para la cual debe completarse.

El tamaño de cada unidad, el tiempo de procesamiento de un batch de cierta orden en cada unidad, el factor de tamaño que indica el tamaño requerido en cada etapa para producir una unidad de masa final como así también el porcentaje mínimo de uso en cada unidad son parámetros del problema.

El problema consiste en determinar el número de batches que se deben procesar para satisfacer la demanda de cada orden, las unidades en que se procesa cada batch y realizar el secuenciamiento de los batches calculando el tiempo inicial y final de procesamiento de cada uno de ellos en cada una de las unidades, teniendo como objetivo minimizar el tiempo total requerido para procesar completamente todas las órdenes, es decir, minimizar el makespan. 
La planta opera de forma secuencial, es decir, cada batch debe seguir la misma secuencia a través de todas las etapas de la planta y ser procesado en exactamente una unidad de cada etapa. Cada batch se trata de manera individual durante el procesamiento, no se divide ni se mezcla con otros batches. A partir de las condiciones anteriores surge la propuesta de que durante su procesamiento cada batch sigue un camino pasando por una unidad en cada etapa. De manera que un camino es un conjunto formado por una unidad de cada etapa.

El concepto de camino, que se introduce por primera vez en este trabajo, permite una simplificación importante en la formulación del modelo, ya que al asignar un batch a un camino se está realizando la asignación simultánea del mismo a todas las unidades donde se procesará, a diferencia de las formulaciones previas que asignan los batches a cada unidad de manera individual.

Puesto que son conocidos el volumen de cada unidad, su porcentaje mínimo de ocupación y el factor de tamaño de cada orden en las diferentes etapas, es posible determinar los tamaños mínimo y máximo factibles en cada camino para un batch de cada orden, lo cual permite plantear restricciones para satisfacer la demanda sin tener que determinar explícitamente el tamaño de cada batch, basta con que la suma de los mínimos y máximos admisibles en los caminos a los que se asignan batches de cierta orden sean respectivamente menor y mayor que el tamaño de dicha orden.

Por último, pero no menos importante, dado que se adopta la política de transferencia zero-wait (ZW), es decir, cada batch que ha sido procesado en una etapa debe ser transferido inmediatamente a la siguiente, basta con conocer el tiempo en el cual se comienza a procesar cada batch en el camino al que se asignó para que queden completamente determinados los tiempos en que el batch se procesará en cada unidad del camino y el tiempo final de procesamiento.

Para realizar el secuenciamiento de los batches, en este modelo se utiliza una representación de tiempo discreto, en la cual se propone una grilla equiespaciada de puntos teniendo en cuenta las fechas de emisión y entrega de las órdenes. Para simplificar la representación se supone que el menor de los tiempos de inicio es igual a 0 y los demás tiempos de inicio y fechas de entrega se proponen respecto a dicho tiempo inicial. El paso de la grilla es igual al máximo común divisor entre los tiempos de procesamiento de todas las órdenes en cada una de las unidades. Cada batch es asignado a un único tiempo de inicio en el camino donde se procesa. El secuenciamiento de batches se realiza de manera implícita imponiendo que en cada unidad y en cada punto de la grilla de tiempos se puede procesar como máximo un batch; esto evita el solapamiento de batches en cada unidad. El modelo determina únicamente el tiempo inicial de operación de cada batch en la primera unidad del camino donde se procesa. Por lo tanto, para determinar en qué puntos de tiempo se procesa en las unidades del camino que no pertenezcan a la primera etapa, deben tenerse en cuenta el tiempo de inicio y los tiempos de procesamiento en las unidades previas del camino.

El objetivo es minimizar el makespan (MK), es decir el máximo tiempo requerido para procesar todas las órdenes.

Ejemplo ilustrativo: se presenta un ejemplo que permite mostrar tanto la capacidad para generar una solución para el problema como la performance computacional del modelo desarrollado en este trabajo. Se considera una planta batch de dos etapas, con tres unidades en cada una de ellas, operando en paralelo y fuera de fase. La estructura, como así también las capacidades máximas de las unidades, son conocidas, y se ilustran en la Figura 1.

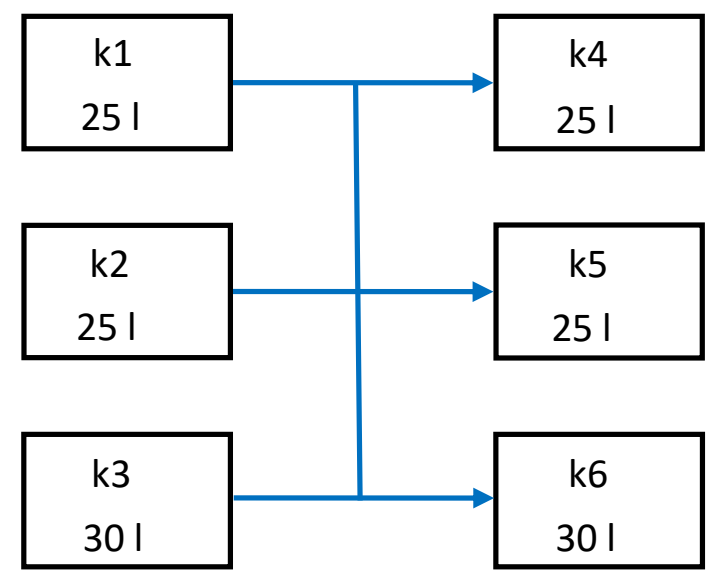

Figura1: Estructura de la planta 
De acuerdo a la estructura de la planta, es posible definir 9 caminos diferentes. Para simplificar, en este ejemplo, se consideran los factores de tamaño iguales a uno para todas las órdenes y todas las etapas, mientras que el porcentaje mínimo de ocupación es del $70 \%$ en cada unidad. Teniendo en cuenta estas consideraciones, es posible determinar los tamaños mínimos y máximos admisibles para cada batch en cada camino, independientemente de la orden. Las unidades que componen cada camino como así también los tamaños mínimo y máximo en cada uno de ellos se muestran en la Tabla 1.

\begin{tabular}{|c|c|c|c|c|}
\hline \multirow{2}{*}{ Camino } & \multicolumn{2}{|c|}{ Unidad de cada etapa que compone el camino } & \multirow{2}{*}{$B_{r}^{\min }$} & \multirow{2}{*}{$B_{r}^{\max }$} \\
\cline { 2 - 3 } & Etapa 1 & Etapa 2 & 17.5 & 25 \\
\hline $\mathrm{r} 1$ & $\mathrm{k} 1$ & $\mathrm{k} 4$ & 17.5 & 25 \\
\hline $\mathrm{r} 2$ & $\mathrm{k} 1$ & $\mathrm{k} 5$ & 21 & 25 \\
\hline $\mathrm{k} 3$ & $\mathrm{k} 1$ & $\mathrm{k} 6$ & 17.5 & 25 \\
\hline $\mathrm{r} 4$ & $\mathrm{k} 2$ & $\mathrm{k} 4$ & 17.5 & 25 \\
\hline $\mathrm{r} 5$ & $\mathrm{k} 2$ & $\mathrm{k} 6$ & 21 & 25 \\
\hline $\mathrm{r} 6$ & $\mathrm{k} 2$ & $\mathrm{k} 4$ & 21 & 25 \\
\hline $\mathrm{r} 7$ & $\mathrm{k} 3$ & $\mathrm{k} 5$ & 21 & 25 \\
\hline $\mathrm{r} 8$ & $\mathrm{k} 3$ & $\mathrm{k} 6$ & 21 & 30 \\
\hline $\mathrm{r} 9$ & $\mathrm{k} 3$ & & & \\
\hline
\end{tabular}

Tabla 1: Definición de los caminos y tamaños admisibles para los batches

La planta debe procesar un conjunto de diez órdenes $\{\mathrm{d} 1, \mathrm{~d} 2, \ldots, \mathrm{d} 10\}$, cuyas demandas, fechas de emisión y límite de entrega, y tiempos de procesamiento se presentan en la Tabla 2.

\begin{tabular}{|c|c|c|c|c|c|c|c|c|c|}
\hline \multirow{2}{*}{ Orden } & \multirow{2}{*}{$Q_{d}(\mathrm{~kg})$} & \multirow{2}{*}{$R D_{d}$} & \multirow{2}{*}{$D D_{d}$} & \multicolumn{6}{|c|}{ Tiempos de procesamiento (hs) } \\
\cline { 5 - 10 } & & & & $\mathrm{k} 1$ & $\mathrm{k} 2$ & $\mathrm{k} 3$ & $\mathrm{k} 4$ & $\mathrm{k} 5$ & $\mathrm{k} 6$ \\
\hline $\mathrm{d} 1$ & 75 & 0 & 20 & 4 & 3 & 4 & 4 & 3 & 4 \\
\hline $\mathrm{d} 2$ & 60 & 0 & 20 & 4 & 3 & 3 & 4 & 4 & 3 \\
\hline $\mathrm{d} 3$ & 45 & 0 & 20 & 3 & 4 & 5 & 3 & 4 & 5 \\
\hline $\mathrm{d} 4$ & 60 & 0 & 30 & 4 & 3 & 5 & 3 & 4 & 4 \\
\hline $\mathrm{d} 5$ & 80 & 0 & 30 & 2 & 4 & 3 & 2 & 4 & 3 \\
\hline $\mathrm{d} 6$ & 65 & 10 & 30 & 3 & 4 & 5 & 3 & 4 & 5 \\
\hline $\mathrm{d} 7$ & 80 & 10 & 35 & 4 & 5 & 4 & 4 & 5 & 3 \\
\hline $\mathrm{d} 8$ & 45 & 10 & 35 & 4 & 3 & 5 & 4 & 3 & 5 \\
\hline $\mathrm{d} 9$ & 65 & 10 & 35 & 4 & 5 & 5 & 4 & 5 & 3 \\
\hline $\mathrm{d} 10$ & 85 & 10 & 35 & 3 & 2 & 2 & 3 & 2 & 2 \\
\hline
\end{tabular}

Tabla 2: Demandas, fecha de emisión y vencimiento y tiempos de procesamiento de las órdenes

Para la resolución del problema se propone una grilla de tiempos discretos desde 0 hasta 35 o sea, desde 0 hasta la mayor de las fechas de vencimiento, con paso igual a 1 que es el máximo común divisor entre todos los tiempos de procesamiento.

El ejemplo fue implementado en GAMS versión 24.7.4 y resuelto empleando el solver CPLEX 12.6.3, con un procesador Intel Core i7, 3.6 GHz, 8 GB de RAM. El modelo consta de 7239 ecuaciones, 5454 variables discretas y 1 variable continua correspondiente al makespan. La obtención del óptimo global demandó un tiempo de CPU de 70 segundos.

En la solución óptima el makespan resulta ser 49 horas. En la Tabla 3 se resumen los resultados obtenidos, indicando los batches en que se divide cada orden, los caminos en que se procesa cada batch y los tiempos iniciales y finales de procesamiento de los mismos, a fin de satisfacer los requerimientos de demanda en el mínimo tiempo total de procesamiento. Como puede observarse, es necesario procesar 26 batches para satisfacer la demanda de las 10 órdenes. Las órdenes d2, d3 y d8 requirieron de dos batches cada una, mientras que las restantes han sido satisfechas empleando tres batches. Cada orden satisface su fecha de emisión y límite de entrega. 


\begin{tabular}{|c|c|c|c|c|}
\hline Orden & Batches & Camino & Tiempo de inicio (hs) & Tiempo final (hs) \\
\hline \multirow{3}{*}{ d1 } & b1 & $\mathrm{r} 5$ & 0 & 6 \\
\hline & b2 & $\mathrm{r} 5$ & 3 & 9 \\
\hline & b3 & r5 & 6 & 12 \\
\hline \multirow{2}{*}{$\mathrm{d} 2$} & b1 & r9 & 3 & 9 \\
\hline & b2 & r9 & 6 & 12 \\
\hline \multirow{2}{*}{ d3 } & b1 & $\mathrm{r} 1$ & 4 & 10 \\
\hline & b2 & $\mathrm{r} 1$ & 7 & 13 \\
\hline \multirow{3}{*}{$\mathrm{d} 4$} & b1 & $\mathrm{r} 4$ & 16 & 22 \\
\hline & b2 & $\mathrm{r} 4$ & 19 & 25 \\
\hline & b3 & $\mathrm{r} 5$ & 9 & 16 \\
\hline \multirow{3}{*}{ d5 } & b1 & $\mathrm{r} 1$ & 0 & 4 \\
\hline & b2 & $\mathrm{r} 1$ & 2 & 6 \\
\hline & b3 & r9 & 0 & 6 \\
\hline \multirow{3}{*}{ d6 } & b1 & r1 & 10 & 16 \\
\hline & b2 & $\mathrm{r} 2$ & 13 & 20 \\
\hline & b3 & $\mathrm{r} 4$ & 12 & 19 \\
\hline \multirow{3}{*}{$\mathrm{d} 7$} & b1 & r7 & 21 & 29 \\
\hline & b2 & r8 & 16 & 25 \\
\hline & b3 & r9 & 12 & 19 \\
\hline \multirow{2}{*}{$\mathrm{d} 8$} & b1 & r5 & 22 & 28 \\
\hline & b2 & $\mathrm{r} 5$ & 25 & 31 \\
\hline \multirow{3}{*}{ d9 } & b1 & r3 & 16 & 23 \\
\hline & b2 & r3 & 20 & 27 \\
\hline & b3 & r3 & 25 & 32 \\
\hline \multirow{3}{*}{$\mathrm{d} 10$} & b1 & r7 & 27 & 32 \\
\hline & b2 & r9 & 10 & 14 \\
\hline & b3 & $\mathrm{r} 9$ & 25 & 29 \\
\hline
\end{tabular}

Tabla 3: Batching y scheduling óptimo de las órdenes

En la Figura 2, se ilustra el diagrama de Gantt chart de la solución óptima.

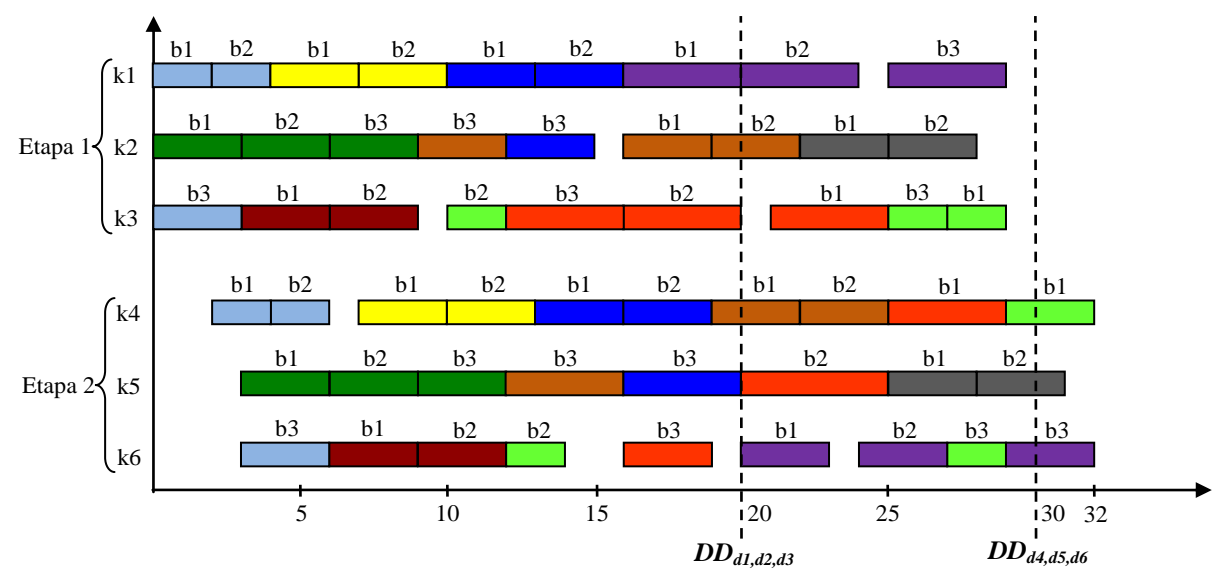

Órdenes

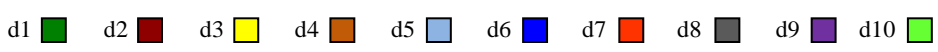

Figura 2: Diagrama de Gantt chart 


\section{Conclusiones}

El modelo desarrollado en este trabajo permite resolver simultáneamente los problemas de batching y scheduling minimizando el tiempo total de procesamiento, proporcionando el número de batches en que se divide cada orden, las unidades en que se procesa cada batch y sus tiempos inicial y final de procesamiento. La nueva representación para decidir el procesamiento de las órdenes, basada en caminos de producción, y la definición de tiempos discretos, han demostrado ser computacionalmente eficiente, permitiendo resolver problemas de considerable tamaño en tiempos reducidos. De este modo, se proporciona una herramienta eficiente para optimizar la operación de una planta batch multiproducto.

\section{Referencias}

Castro, P. M., Grossmann, I. E., \& Zhang, Q. (2018). Expanding scope and computational challenges in process scheduling. Computers \& Chemical Engineering, 114, 14-42.

Fumero, Y., Corsano, G., \& Montagna, J. M. (2014). Simultaneous batching and scheduling of batch plants that operate in a campaign-mode, considering nonidentical parallel units and sequence-dependent changeovers. Industrial \& Engineering Chemistry Research, 53(44), 17059-17074.

Harjunkoski, I., Maravelias, C. T., Bongers, P., Castro, P. M., Engell, S., Grossmann, I. E., ... \& Wassick, J. (2014). Scope for industrial applications of production scheduling models and solution methods. Computers \& Chemical Engineering, 62, 161-193.

Lee, H., \& Maravelias, C. (2017a). Discrete-time mixed-integer programming models for short-term scheduling in multipurpouse enviroments. Computers \& Chemical Engineering, 107, 171-183.

Lee, H., \& Maravelias, C. (2017b). Mixed-integer programming models for simultaneous batching and scheduling in multipurpose batch plants. Computers \& Chemical Engineering, 106, 621-644.

Maravelias, C. T. (2012). General framework and modeling approach classification for chemical production scheduling. AIChE Journal, 58(6), 1812-1828.

Méndez, C. A., Cerdá, J., Grossmann, I. E., Harjunkoski, I., \& Fahl, M. (2006). State-of-the-art review of optimization methods for short-term scheduling of batch processes. Computers \& Chemical Engineering, 30(6-7), 913-946.

Merchan, A. F., Lee, H., \& Maravelias, C. T. (2016). Discrete-time mixed-integer programming models and solution methods for production scheduling in multistage facilities. Computers \& Chemical Engineering, 94, 387-410.

Prasad, P., \& Maravelias, C. T. (2008). Batch selection, assignment and sequencing in multi-stage multi-product processes. Computers \& Chemical Engineering, 32, 1106-1119.

Sundaramoorthy, A., \& Maravelias, C. (2008a). Simultaneous batching and scheduling in multistage multiproduct process. Industrial \& engineering chemistry research, 47(5), 1546-1555.

Sundaramoorthy, A., \& Maravelias, C. (2008b). Modeling of storage in batching and scheduling of multistage process. Industrial \& engineering chemistry research, 47(17), 6648-6660.

Sundaramoorthy, A., Maravelias, C. T., \& Prasad, P. (2009). Scheduling of multistage batch processes under utility constraints. Industrial \& engineering chemistry research, 48, 6050-6058. 